\title{
Saturable absorber mirrors for ytterbium mode-locked femtosecond lasers
}

\author{
Łukasz Zinkiewicz, ${ }^{* 1}$ Michał Nawrot, ${ }^{1}$ Agata Jasik, ${ }^{2}$ Iwona Pasternak ${ }^{3}$, and Piotr Wasylczyk ${ }^{1}$ \\ ${ }^{1}$ Photonic Nanostructure Facility, Faculty of Physics, University of Warsaw, Hoża 69, 00-681 Warszawa, Poland, \\ ${ }^{2}$ Institute of Electron Technology, Aleja Lotników 32/46, 02-662 Warszawa, Poland \\ ${ }^{3}$ Institute of Electronic Materials Technology, Wolczynska 133, 01-919 Warsaw, Poland
}

Received September 24, 2013; accepted March 26, 2014; published March 31, 2014

\begin{abstract}
We present a femtosecond, diode-pumped Yb:KYW laser configured with a range of saturable absorber mirrors, both commercially available and state-of-the-art structures. The laser performance is characterized for different output couplers (OC) transmission - for the typically used OC transmission between $0.5-10 \%$ the laser power increases with the increasing transmission but the spectrum gets narrower at the same time, resulting in longer pulses and the laser pulse peak power having a maximum.
\end{abstract}

In the last decade new femtosecond lasers, in particular based on Yb:crystal media [1-5] have been developed as robust, compact light sources, capable of delivering highpower femtosecond pulses in the near infrared. Dispersion management in Yb:KYW lasers, based on dispersive (chirped) mirrors has improved the lasers stability compared to the early prism-based designs. The cavity dispersion compensation with high negative group velocity dispersion (GVD) dielectric [6] or semiconductor [7] multilayer mirrors has been demonstrated, including the cavity with a single, extremely large negative GVD semiconductor reflector [8]. Saturable absorber mirrors (SAMs) supporting the Kerr effect mode-locking have further enhanced the stability and allowed for self-starting pulsed laser operation [9-10].

In this letter we report the study of several saturable absorber mirrors in a femtosecond Yb:KYW oscillator. The laser performance was measured for both commercially available as well as state-of-the-art structures, including a graphene-coated dielectric reflector, which is, to our knowledge, the first demonstration of an $\mathrm{Yb}: \mathrm{KYW}$ laser with a graphenebased SAM.

The Yb:KYW femtosecond laser had a 1mm, Brewstercut $\mathrm{Yb}: \mathrm{KY}\left(\mathrm{WO}_{4}\right)_{2}$ crystal doped at 10 at.\% (Eksma) in a Z-folded, astigmatically compensated cavity, pumped with a 980nm, 550mW CW laser diode (LC96UF80, Bookham) (see Fig. 1). The cavity was terminated by a saturable absorber mirror at one end and dispersion compensation was provided by a set of three dielectric

\footnotetext{
*E-mail: lukasz.zinkiewicz@fuw.edu.pl
}

mirrors (Layertec), accounting for the total GVD of -2500 $\mathrm{fs}^{2} @ 1030 \mathrm{~nm}$.

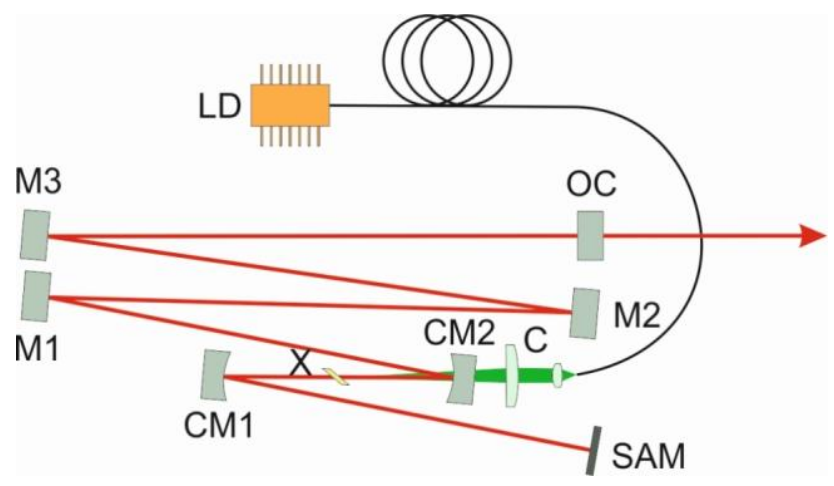

Fig. 1. Femtosecond diode-pumped Yb:KYW laser with a saturable absorber mirror (SAM), where: LD - fibre coupled laser diode, $\mathrm{C}$ collimating ( $\mathrm{f}=15 \mathrm{~mm}$, aspheric) and focusing $(\mathrm{f}=63 \mathrm{~mm}$, plano-convex)

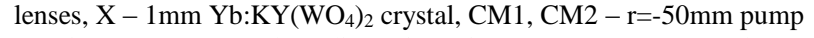
mirrors, M1-M3 - plane dispersive mirrors, OC - output coupler (various transmissions - see the text for details).

We investigated a series of laser cavity configurations, arranging all possible combinations of five different saturable absorber mirrors - see Table 1 - and six output coupler mirrors with transmissions of $0.5,1.5,2.5,5,8$ and $10 \%$. Three of the SAMs were commercially available semiconductor mirrors from Batop GmbH [11]. One (IET SAM) was a 24-pair AlAs/GaAs distributed Bragg reflector with an extended top GaAs layer. The $\mathrm{In}_{0.26} \mathrm{GaAs}$ absorber layer was capped with $5 \mathrm{~nm}$ of $\mathrm{GaAs}$ and the structure was topped by a quarter-wavelength $\mathrm{SiO}_{2}$ layer [12]. The last $\mathrm{SAM}$ was a graphene layer produced by chemical vapor deposition on a copper foil and transferred onto a dielectric, high-reflective (HR), dispersive $\left(\mathrm{GVD}=-250 \mathrm{fs}^{2}\right)$ mirror in the process described in detail in [13]. Table 1 lists the maximum continuous wave $(\mathrm{CW})$ laser power measured with a $1.5 \%$ output coupler for different SAMs, plus the laser without SAM (cavity terminated with a dielectric HR mirror). 
Table 1. Measured CW laser output power with different saturable absorber mirrors and a $\mathrm{T}=1.5 \%$ output coupler. The bold lines indicate the configurations for which mode-locked operation was achieved. For the IET SAM, only a mode-locked operation was possible.

\begin{tabular}{|l|c|}
\hline \multicolumn{1}{|c|}{ Saturable absorber mirror } & $\begin{array}{c}\text { CW laser output } \\
\text { power }(\mathrm{mW})\end{array}$ \\
\hline Batop SAM-1040-2-500fs & $\mathbf{5 5}$ \\
\hline Batop SAM-1040-0.7-500fs & 76 \\
\hline Batop SAM-1040-2-1ps & 50 \\
\hline IET & $\mathbf{5 4}$ (ML) \\
\hline Single graphene layer & $\mathbf{4 3}$ \\
\hline No SAM & 82 \\
\hline
\end{tabular}

Neither for the two (0.7-500fs and 2-1ps) Batop SAMs, nor for the no-SAM configuration was the mode-locked operation of the laser achieved, even with a lowtransmission $(\mathrm{T}=1.5 \%)$ output coupler, and these mirrors were not investigated further. Other SAMs were tested with different output couplers so as to verify the presence of mode-locking with various intracavity pulse power. In terms of the average laser output power, all the three SAMs preformed comparably.

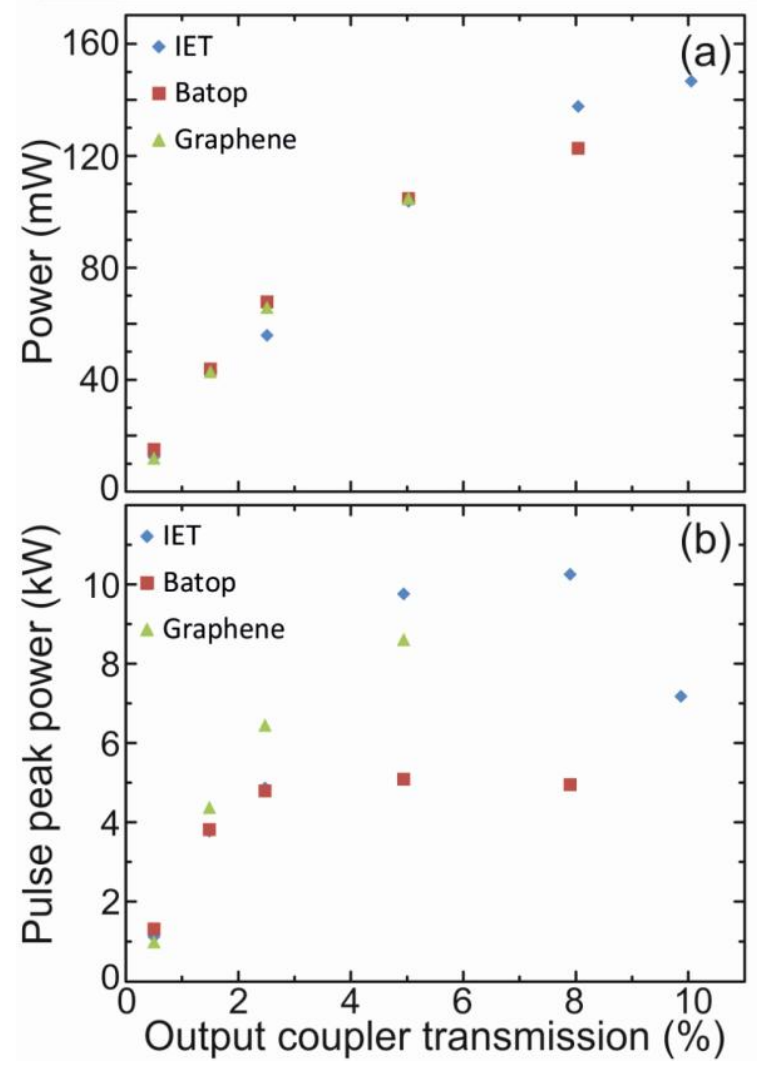

Fig. 2. Measured laser average power (a) and pulse peak power (b) in the mode-locked regime versus the OC transmission for three different SAMs: Batop (red squares), IET (blue diamonds) and graphene (green triangles) - no mode-locking is observed above a certain OC transmission, characteristic for each SAM.
In Fig. 2 the pulse peak power and the laser average output power in the mode-locked regime are plotted. The latter was increasing with the output coupler transmission for all SAMs - even for the highest OC transmission there was hardly any sign of approaching saturation. No modelocking was observed for the Batop mirror with $\mathrm{T}=10 \%$ $\mathrm{OC}$, nor for the graphene SAM and OC with transmission above 5\%, while the IET semiconductor SAM mirror still supported femtosecond pulses with the average power of nearly $150 \mathrm{~mW}$ for the $10 \%$ OC. The pulse peak power was rising first with increasing output coupler transmission to reach the maximum for $\mathrm{T}=5 \%$ and then was saturated (Batop) or decreased (IET). The highest peak powers, reaching $10 \mathrm{~kW}$, were observed for the IET SAM, doubling the performance of the commercial mirror.

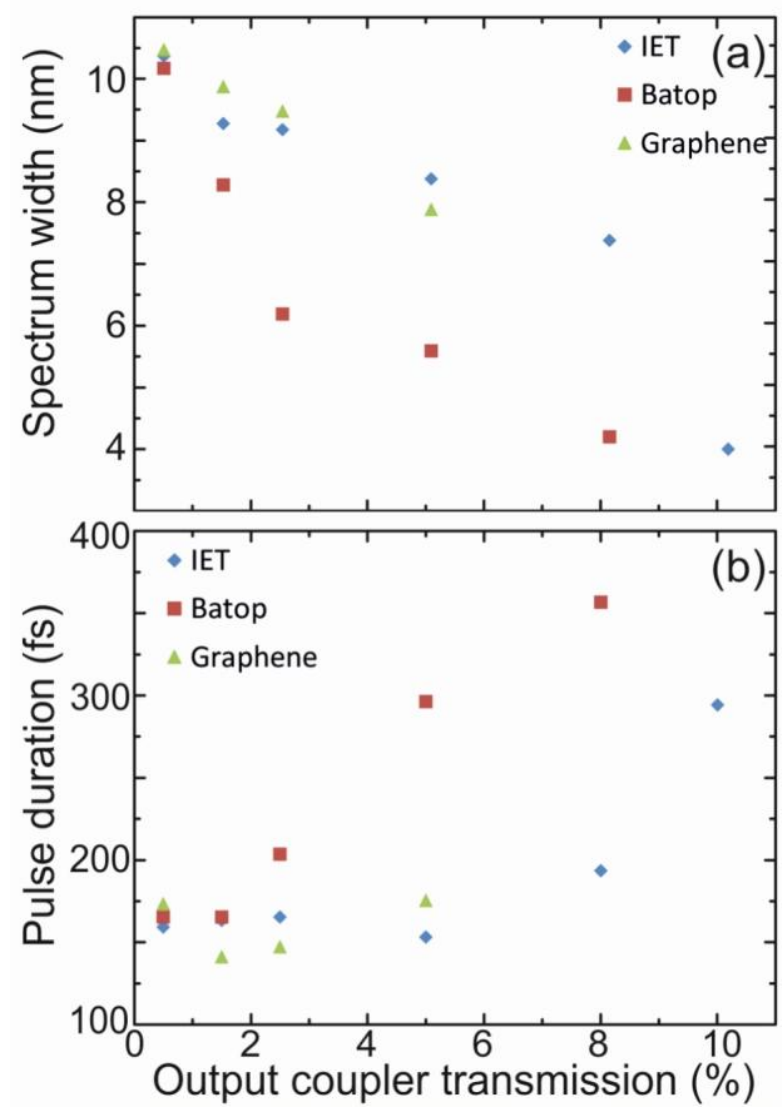

Fig. 3. Measured laser spectrum width FWHM (a) and the pulse duration (FWHM, assuming Gaussian envelope) (b) for different output couplers and three different SAMs: Batop (red squares), IET (blue diamonds) and graphene (green triangles). The pulse durations were retrieved from interfereometric autocorrelation measurements.

Apart from the laser power, the laser spectra and pulse durations were measured for different cavity configurations. In all cases, the spectrum width was decreasing monotonically with the increasing output 
coupler transmission (see Fig. 3 a). This in turn resulted in increasing laser pulse duration - see Fig. 3 b. For lowtransmission OCs, the saturation of the pulse length was observed - the shortest measured pulse FWHM (assuming Gaussian envelope) was around 150fs, which was probably limited by the non-optimised cavity dispersion. The shortest pulses are measured with the graphene SAM, possibly also due to the additional $-250 \mathrm{fs}^{2}$ GVD of the mirror (other SAMs have measured GVD close to zero). The semiconductor Batop mirror generally supports a narrower spectrum, resulting in longer pulses at comparable output powers.
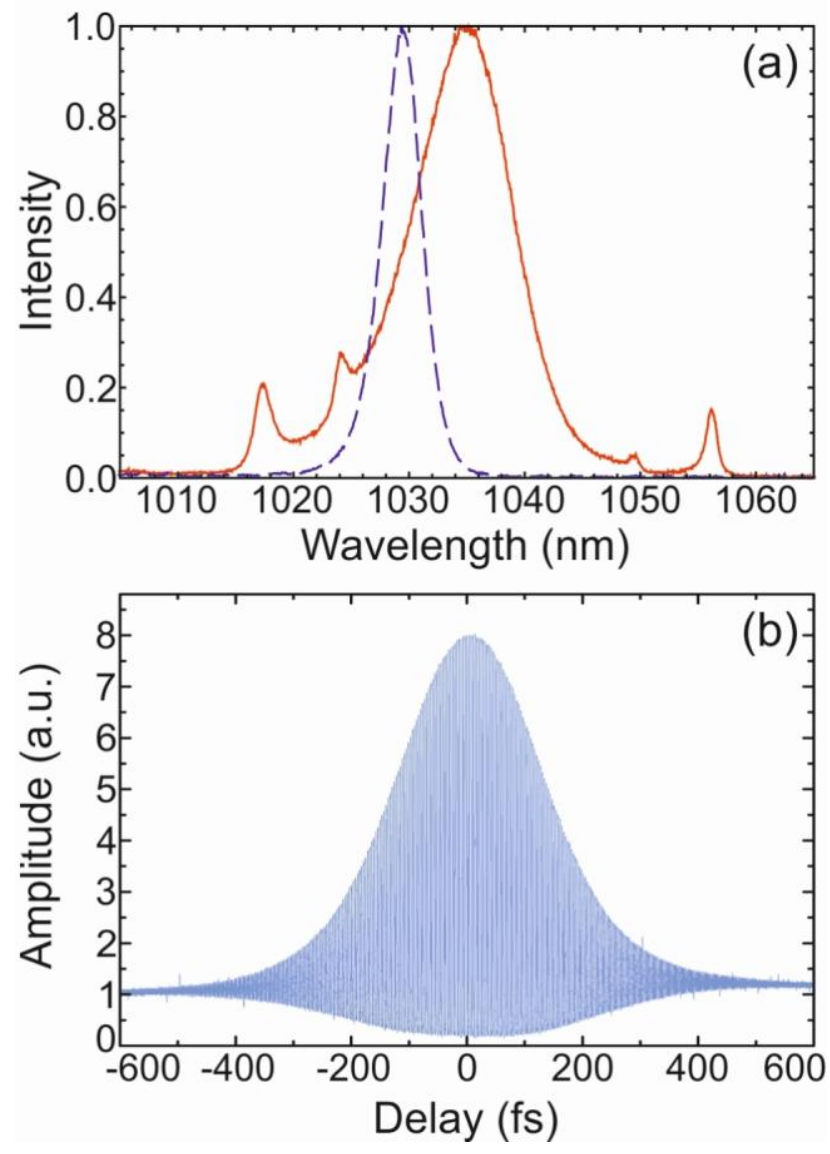

Fig. 4. With the IET SAM the $\mathrm{T}=0.5 \%$ OC supports a wide ( $\mathrm{FWHM}=10.4 \mathrm{~nm}$ ) spectrum (red solid line), while for $\mathrm{T}=10 \%$ only 4.0nm (FWHM) is emitted (blue dashed line) in the mode-locked regime (a). Measured interferometric autocorrelation of a $150 \mathrm{fs}$ pulse (Gaussian envelope), corresponding to the broad spectrum above (b).

Figure 4 shows typical laser spectra with the IET SAM and both low-transmission $(\mathrm{T}=0.5 \%)$ and hightransmission ( $\mathrm{T}=10 \%$ ) OCs. The measured interferometric autocorrelation of a 150fs pulse (FWHM for a Gaussian envelope), indicates a structureless pulse with a timebandwidth product of 0.46 .

In conclusion, we have presented a systematic study of a femtosecond Yb:KYW laser with different saturable absorber mirrors and different output couplers. We have demonstrated that a femtosecond $\mathrm{Yb}: \mathrm{KYW}$ laser can be mode-locked with a graphene-based SAM. For the OC transmissions typically used in ytterbium fs oscillators, the laser power increases monotonically with increasing transmission. This is, however, at the price of narrowing the laser spectrum, thus increasing the pulse duration - the pulse peak power reaches the maximum for OCs with a transmission around 5-8\%.

This work has been supported by the National Science Centre (NCN, Poland) within the DEC2012/05/E/ST3/03281 and 3606/B/T02/2009/36 and by the National Centre for Research and Development (NCBiR) 02-0009-10/2011 grants funds. Partial support by ERDF within the POIG.02.01.00-14-122/09-00 is also acknowledged.

\section{References}

[1] C. Hönninger, R. Paschotta, M. Graf, F. Morier-Genoud, G. Zhang, M. Moser, S. Biswal, J. Nees, A. Braun, G.A. Mourou, I. Johannsen, A. Giesen, W. Seeber, U. Keller, Appl. Phys. B 69, 3 (1999).

[1] A. Lagatsky, C. Brown, W. Sibbett, Opt. Expr. 12, 3928 (2004).

[2] H. Liu, J. Nees, G. Mourou, Opt. Lett. 26, 1723 (2005).

[3] P. Wasylczyk, C. Radzewicz, Laser Phys. 19, 129 (2009).

[4] S. Meyer, J. Squier, S. Diddams, Eur. Phys. J. D 48, 19 (2008).

[5] D. Kopf, G. Zhang, R. Fluck, M. Moser, U. Keller, Opt. Lett. 21, 486 (1996).

[6] R. Paschotta, G. Spuhler, D. Sutter, N. Matuschek, U. Keller, M. Moser, R. Hovel, V. Scheuer, G. Angelow, T. Tschudi, Appl. Phys. Lett. 75, 2166 (1999).

[7] A. Jasik, P. Wasylczyk, M. Dems, P. Wnuk, A. Wójcik-Jedlińska, K. Reginski, Ł. Zinkiewicz, K. Hejduk, Laser Phys. Lett. 10, 085302 (2013).

[8] A. Schmidt, S. Rivier, W. Cho, J. Yim, S. Choi, S. Lee, F. Rotermund, D. Rytz, G. Steinmeyer, V. Petrov, U. Griebner, Opt. Expr. 17, 20109 (2009).

[9] E. Rafailov, S. White, A. Lagatsky, A. Miller, W. Sibbett, D. Livshits, A. Zhukov, V. Ustinov, IEEE Photon. Technol. Lett. 16, 2439 (2004).

[10] http://www.batop.de/products/saturable-absorber/saturable-absorbermirror/saturable-absorber-mirror-1040nm.html

[11] A. Jasik, J. Muszalski, J. Talalaev, M. Kosmala, K. Pierściński, M. Bugajski, J. Appl. Phys. 109, 053101 (2009).

[12] G. Sobon, J. Sotor, I. Pasternak, K. Grodecki, P. Paletko, W. Strupinski, Z. Jankiewicz and K. Abramski, J. Lightw. Technol. 30, 2770 (2012). 